\title{
A "Estrutura Matemática" da Natureza e da Ciência
}

\author{
A.M. AMORIM DA COSTA*
}

\section{MATEMÁTICA E EXPERIÊNCIA}

Quando se pretende [azer o elenco dos factos ou das liguras que mais marcaram uma determinada éfuca, muito dificimentc " mesmo facto ou a mesma ligura merecerá consenso unẩ เฉime de qự seja chamado a (azê-10. Com naturalidadc, dilerentes pontos de vista e difcruntes critérius de valuraçâo levan a difcrentes catalogaçōes. Ao terminar o segund»s miléniou da ura cristã tom que vivemos, um pouco por toda a parle, aparcceram opiniōes variadas sobrc a acontecimento e sobre a figura que mais o terão marcado. No emaranhado da natural falta de comsenso, a Revoluçăn Científica ocorrida ao longo dos séculos XVI e XVII e, no contexto dela. os nomes de L. Da Vinci (14521519), N. Copérnico (1473-1543), I. Kepler (157/-1630), Galileu-Galilei (1564-1642) e I. Newton (16421727 ) recolhem of favoritismu da maioria dos analistas.

Reportando-nos à figura de Galileu, gostaríamos de notar aqui a importância que ele atribuía à matemálica no estudo das ciências e. sobretudo, a filosolia en que suporlava essa importância.

É justannente famosa a ufuestăo 6 do seu tratado O Ensuiador, escrito cm 1623: "a filosolia do Universo. esse grandissimo livro que continuamentc está aberto em fremte de nossos olhos, nâo se pode entender sem primeiro se conhecer a linguagt'm e us caracteres ém que está escrita. A sua linguagem é uma linguagem malemática em que os caractercs sẫo os rriângululıs, os círculus e demais figuras geométricas, sctn o conhecimeno dos quais en impossivel sntender uma sí das suas palavras" 11]. De acorư com Galileu, é preciso conhecer ben a ciência matenálica para poder conliecer a Nalureza; c só é possível intcrrogá-la devidamente usando a linģuagem matemálica. É experimentando, com experiências reais e experiĉncias imaginadas que o cientista interroga a Natureza. Consequentemente. a terria matemática precedt' a txperiência. Nesta concepgão de Gałileu ter1contranus, pela primeira vez na história do pensameino bumano, a idéia da física malemática, ou, cm alimação mais unlática, a idêia do malcmatismun [isico [2]

O plapel das matemáticas $n$ n desenvolvimento da ciência lísica não cra, an tempo de Galilcu, um problema novo. A sula consideração e discussão, no Ocidente, constavaln de mais que uma das correnres das Escolas da Alttiga Grécia. O própro Galifeu estava consciemc disso c tinha por certo tralar-se duma questão que lora asstunto fundamental de disputa cntre Aristótcles e Platão. Disso mesmo nus dá conta no seu Dislogo sobre or Dois Prinifutis Sistemas to Munds, escrito cm 1632, onde, Salviati, o seu porta voz, afirma gute "as concluso. es matemáticas sāo exactamente as mesmas no abstracto e no concreto", relumando a posição de simñlício que defondenda A ristótules contra Platăo, considerava que "as subuilezas matemáticas apenas funcionam muito bem no abstracto. mas năo quando sc tenta aplicá-las à matéria física sensivel " [3]. F mais adiante: "não há outra disputa duน tenha dado lugar a tantas especula.

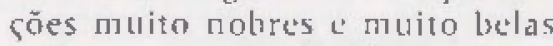
(...) como a questāu sobre se o uso das matemátícas na ciência lísica, encuanto instrumento de prova e tcrmo médio da demonstraçăo é upmetuno ou inoportuno; isto é, se c.le nos traz alguma verdade, ou, se. pelo contrário é prejudicial c perigoso. Com eleito. Platão acreditava que as malumálicas eram muito particularmente acomodadas às especulaçóes físicas; e ca por isso que ele recorreu muitas vizes a clas para explicar os mistérios fisicos. Mas Aristóteles parece ter tido um scrtimento intciramente opms10. č atribuía os crros de Platāo a unI demasiado amor pelas matemálicas" [4].

Como nota $A$. Koyré, a diterenร̧a entre os sequazes de Aristóteles c os sçuluazes de Platāo neste assumu nāo é de modo algum. o problema da ccrleza, mas sim o da realidade: nàı ć de modo algum o emprego das matcmáricas na ciência física, mas simn o do sell papel na e para a mópria estrutura da ciência, isto ć, da própria realidade [5].

Rçnortando à estrutura da ciềncia c, conșequentemementc. da própria realidade, o matematismo lísicu de Galileu mão é. todavia, de moto algum, a defesa da matematizaçäu da natureza, yue outra coisa se กã pode concluir da sua afirmação explicita: "nas demonstraçôes naturais. não se deve procurar a exactidã matemática" 16]. pois yue a realidade física - quantitaliva e imprecisa nảo se molda por si frópria à rigidez de nuşīes matemáticas. A maséria natural nunco encarna [ormas precjsas. c as formas nunca a informam perfeitamente; sobra strupre "jogus e. portanto, a querer matematizar a natureza não se chega a lado nenhum. No mundo real - o mundo lí* sico - nāo há recclas, nem planos, nem triângulos, nem es[eras; os corpos do mundo material nă possuem as formas regulares da geometria. As lcis geomcétricas naa thes podcm. portanio, ser aplicadas. As leis matemáticas são, para a rcalidade lísica, leis aproximadas: os seres físicos "imitam" e se "aproximam" dos seres geumérícos [7]. Na sua essê̂]. cia ûltima, o real é matemálico, n mesumo dizer. pode traduzir-se em te'rmos matcmáticos: mas a matemática não é o rexal. $\Lambda$ forma geométrica ć homogúnta à matéria e, portanto). as leis geométricas tền um valor réal e dominam a ciência que as ten por objecto. mas não curstituem elas própria a realidadc. A teoria mate. mática. cumo acima ficoll escrito, precede a cxperiencia porque é no scu formalismo que esta se objeciva: mas a objectividade da experiência năo se consulsstancia no formalismo que a suporta.

\section{A "REALIDADE" DAS ORBITAIS MOLECULARES}

As consideraçōes que acabatums de exprimir por réleréncia sumária 
ao matematismo físicn de Galileu. que lemos comb um dos mais marcantes acontecimentos do milénio ora lindo. servem-nos de hase para nos referirmos aqui à polémica recentemente gerada. no dominio da química-física, em torno do carăcter experincutal das orhitais moleculares, nla seụuência da "observaçău" das orbitais d por J.M. Zuo c colaboadores, da Universidade do Estado de Arizona, nos Estados Unidos, cm 1999.

Na capa do seu número de 2 de Selembro de 1999, a revista Nalure deslacava "observadas orbilais", dando realce à comunicaçāo de J. $\mathrm{M}$. 7uo el al. quc trazia publicada nas páginas 49.52 [8]. E ainda antes do se chegar às páginas cm quc a comunicaçāo cra apresentada, na secçào "news and views". Colin J. Humphrcys. do Departamento de Ciência dos Materiais c Mctalurgia, da Universidade de Cambridge (Inglaterra), reforçava a ên[ase contida no título da capa, reterindo que "o formato clássico das orbitais elecurónicas apresentado nos livros de texin loi agora ohscrvado directamente" [9]. Aprescintando expressamente a comunicaçào de J. M. Zuo e colaboradores inserta umas páginas à frente. c. Humphreys refere que estes "usaram uma combinaçāo de dilracção de clectrenes e raios-X nara estudar formalo e ligaça dos átumos de cobre no úxido de cohrc. Esic esiudo revelou experimentalmcite, pela primeira vez, o impressionante formaus de algumas das orbitais electrónicas". E sublinha: "a comunicaçă de Zıo e colaboradores é notável porque a qualidade dos mapas de densidade de carga que aprescmta permite, pela primeira vez na histó. ria, uma folografia direcia e experimental do formato complexo da orbital $d_{z}{ }^{2}, "$

Na análise pormenorizada dos dados que oltiveram pelo já teferido método aplicado ao estudo da ligação Cu-Cu no Cu그. Zuo e colāboradores mostram-se de facto maravilluados pela nolável correspondência enire os mapas de densidade elecirónica que ohtiveram experimcotal- menlc e os diagramas clässicus da or bilais $\mathrm{d}_{z^{2}}$ que qualquer Manual de Quimica apresenta: uma forte distorçãn nāo-eslérica à volta dos átomos de cobre. com o forman caracterísi ico das orbilais d, e um excesso dc carga па região intersticial. O timilo da comunicaçāo é u indicador mais claro do sentido lísico que us autores atribuem à interprecaçān dos dados ohtidos experimentalmente na sua relaçào com a naıureza das orbitais moleculares envolvidas na ligação química $\mathrm{cm}$ apreço: "direct observation of d-orbital boles...".

O impacio deste irabalho foi grande, tendo sido um dos considerados na elahoraçăo dẹ unı lista dos cinco acontecimentos mais significativos no domínio da Quimica. em 1999 [10]. A validade da interpretacāo que os autures deram ans resultados obtidos fol sublinbada com grandc ênfase em várias récerências. Nomeadamente, em página da Internet, o assunto [oi apresentado nos seguintes termus: "do há munto que a ideia das orhitais se' revclou de grande utilidade para descrever ma-

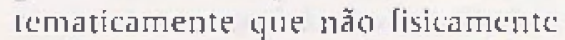
os átomos e as suas interacçōes. Agora tudo mudou. Investigadores da Universidade do Fstado do Arizona publicaram recentemente na Naare as primeiras imagens verdadciras de urhitais atómicas no $\mathrm{C}_{1} \mathrm{O}^{-}[\mathrm{ll}]$. De igual modu, M. Jacoty, no Chem. Eng. News, declarava: "lembram-se daquela orbilal d relerida nos Manuais de Quimica parecida com um otto uridimemsional com $\mathrm{mm}$ donu em torno da sua parte central? Bent, cla acaha de ser observada experimen. talmente por Ciemtistas da Universidadc do Estado do Arizona [12].

Esta interprelaçăo apresentação dos resultados experimentais do grupo de J.M. 7.10, sublinhando uma observaço direta de uma contidade definida e iida com soluçāo tcórica de um formalismo matemático, de imediato susciton a opusiça em alguns meios acadénicus, mantendo viva a questāo do realismo cicntifico de muila da terminologia usada pur químicos c. lísicus.

Uma das posiçoes mais claras no quadro desta nolémica foi a de Eric Scerri do Deparamento de Quimica e Binquintica da Universidade da Califórnia (Lus Angeles). Editor Principal das revistas Fondations of $\mathrm{Cht}$ mistry e' Hyle, duas revistas dedicadas a aspecios filosóficos, históricos, educacionais, culturais e concepuais ro dominjo da Quimica. E. Scerrie autor de variados e extcnsos trabalhos em atue por mais de urna ver. tem aflorado o problema do reducionismo e do realismo em Química [13].

Abordando expressamenc a pruhlemática suscilada pelo 1rahallio de J.M. Zuo e colaboradares e pelos comentarius que sohre ele foram sendo publicados, cm artigo publicado tho Journal of Citcm. Eduction de Novembro de 2000 [14]. Scerri í calegóricu: "n que Zuo e colaboradores fizcram foi ajustar dados expertuenlais obtidos por dilracçāo de raios- $X$ e de electrōes a um modelo conhecido por refinamento de multipolo. Este metcodo näu assume qualquer soma lacuual de contribuiçōes atómicas, mas ajusta os dados usando uma expansão en termus de funçỏes radiais mulıiplicadas nor larmónicos esléricus em várins centros. O resultado ú uma dénsidade de carga que : c'ntio comparada contr a densidade de carga que se obtém por sohreposição de comtribuiçōes alómicas supondo qut o composto seja perleitamentc iónico, o mapa que traduz a variaçāo da donsidade de carga em estuda curresponde pois à dilerença ctutce aluste experimental e o ajustc estérico nu puramente iónico. Na generalidade, o resultado das experiEncias deste lipo a sua subscyututc análise a a densidade electrónica 10เa], que pode ser observada direclamente. e éco frequentemente. No caso de cristais moleculares ou de metais, não se assume sequer quc o composto seja iónico.

"Não nego que as lécnicas usadas por Zuo c col. fornç̧am ll ma imagem da densidade elcotrítica tatal 1105 compostos de cobre em questàn. $O$ que questiono é que esia imagem constitua unu ohservaçàn direcia das urbitais electrónicas. (...। 
Nāo se devem conlundir us termos "densidade electrónica" e "orbilal". Cada uın deles tem o seu significado preciso a o de ambos é e deve ser conservado distinto* [15].

Deixando claro que em sua opiniầ, o que Zuo é colabmadores haviam obscrvado directamente está longe dc ser as orbitais d de que falam os Manuais dc Química, neste seu artigo Scerri é peremptrírio: "nāo cstá em causa apenas o taclo que as orbitais näo podem ser observadas directamente: é que pura c' simplesmente, năo podem ser olsservadas. Enquanıo nada há no formalismo da Mecânica Quântica que proíha a observação do átomos fou densidade electrónica), a própria tcoria estabelece que as orbitais nāo sāu observáveis. A tcoria podc estar errada; mas. se csse é o caso, impöe-se encontrar evidência indeptndente que mostre que ela se não aplica à siluação $\mathrm{cm}$ causa" [15].

Esta posição peremptória de Scerri negando toda a possibilidade de observaçāo das orbitais. por não serem estas realidades físicas que possam ser objecto de deltcção experimcntal directa (í nestc sentido que nuste assunto sc deve enrender o processo de observaçăo). é posíção por ele defendida há longos anos como já referimos. E näo está só. No mesmo sentido. réagirant. por exemplo, Spackman et al., da Uniào Internacional de Cristalogra. fia [16] c o grupo de W. H. Schwarz, na Alemanha [17].

E de referir aqui a polémica yue nos princípios da década de 1990 se gerou em torno da natureza da ligaçăo yuimica, envolvendo o próprio Linus Pauling, a partir de um artigo de J. F. Ogitvic do Instiiuto de Ciéncias Atómica e Moleculares da Academia Sinica (Taiwan). nas páginas do Joumal of Chemical Education. em que o sublílulo era "there are no such things as orbjtals" [18]. Neste seu arigo, Ogilvic deixava claro que as orbitais molecularus eram tảo somente objectos de pensamento ("objects of

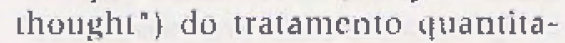
livo e matemático da teoria mecā- nico-quântica aplicado ao estudo da estrutura molecular e das reais propriedades da maléria: tomá-las cumo ruas e fazer delas ponto chave da ciência química é altamentc prejudicial para esta mesma ciência, Defensor de um tratamento do desenvolvimenuo da ciência Química baseado no tsududo cxperimental das substâncias e suas propriedades, no relerido arligo, para Ogilvie a caracterização geral da ligaça química nào precisaría de ir muito além do sabcr-se que ela reflecte forças eléctricas com origem em partículas cléctricas cujas coordenadas e momentos podem ser tratados pela lei de comulação. A consideração da sua naturea em termos de orbitais seria de codo irrelcvantc é irrealista por năo serem elas mais yue arrefactos matmáricos. E nảo é dı artúfacios matemáticos yue podum decorrer propricdadus observávéis de um qualuputr objecto físico [19]. E, referindo-se concretamćnte à csłrutura terlraédrica de moléculas como u melano, alirma tratar-se de pressupostos falaciosos. pois "nem existe hoje, nem nunca existiu qualquer justificaçào yuantitativa experimental ou teririca" que possa comprovar a bibridizaçäo $\mathrm{sp}^{3}$.

Quase dois anos depois, na sua réplica a Ogilvie. Linus Pauling [20] па̃o concorda de modo algum quc sc possa atribuir pouca importância c interesse à caracterizaça possível da ligaçāo química e, mais genericamentc, à tstrutura molecular. Afirma uma vez majs o valor yue continua a atribuir aos muitos 1rabalhos realizados por si e toda члา numerosa plêiade da investigadores. na segunda metade do século $\mathrm{XIX}$ e ao longo de 10 do o século $\mathrm{XX}$. c. em parlicular, a partir da teoria nor si próprio descnvolvida . com início na sua publicaçào "A Narmeă da Ligação Quimion", em 1931 [21]. Mais de sussenta anos volvidos sobre os seus primeiros contribulos para a caracicrizaçào do conceíto da ligação çuímica. reitera toda a sua convicção na validade do mesmo $c$ afirma, novamente, ser esse conceito o mais valioso da ciéncia quínica moderna.

Mas, relativamtente à afirmação substanciata no subeítzlo do artigo de Olgivie, "there are no such things as orbitals", L. Pauling năo conseguiu avançar com outros argumentos yuc não lossem os decorrentes da simples refutação tógica traduzida na afirmaçāo dé que olgìvic ao admitir que as orbitais mais nảo eram que "objectos de pertsamento" estaba a admilir imediatamente quc elas eram alguma coisa". precisamente "objectos de pensamento", pelo que nāu poderia afirmar, sem se contradizer, uratar-se pura e simplesment" de "coisas" que ॥ăo existem.

Deixamos ao léitur ajuizar por si priprio a força dos argumentos exibidos de parte a partc, na cerleza de que nāo falıu guem de imediato tercesse armas de argumentaça ém lavor de qualquuer das partes em conterida, como o deixa claro uma Nota do Editor do Journal of Chemical Education anexa à réplica de L. Pauling au escrito de Olgivie. Por nós voltamos aus escritos de Galileu em torno da estruiura malamática da cièncía. A naturcza está escríta numa linguagem matemática. As rectas, os círculos, os triầngulus e outras figuras geométricas sào os caracteres dessa linguagem que nằ poderemos compreender nem expressar em termos cicrtificos se os nảo conhecermos, ncm considerarmos. Mas dai a defender com Platăo que também o elemtento guométrico é constitutivo de tudo quanto cxiste, parece-nos scr ousadia que só o domínio estritamente filosófico comporta. Nào há ciência scm interpretaçă dos factos observados. Caracreres da linguagem em que a natureza se exprime. as entidades matemálicas são a base da sua interprelaçäo. Todavia, a sua naturcza näo permite que as possamos observar directamente; a sua validadc traduz-sc ent juízos cujo verdadeiro valor é permilicem yue delas sc exuraiam principios gerais das experiincias directas c imedialas yue relacionem os resuladas destas com 
ourros aconıecimentos já observados ou quc poderão vir a ser observadios.

Os quimicos computacionais usem as orbitais e as configuraçōes electrónicas como "fiçōes" matcmáticas. A sua utilização para interpretar c melhor comprcender qujmicamente os lêśmenos observados não pode ser feita atribuindolhe características duma existência definida, como frequentemente parece subjazer ao tratamento dos [cnómenos químicos em que à química quântica é conlerido autêntico estatulo de um axioma com hase no qual se procura construir a ciência química por processo quase-dedulivo em que toda a ênfase ć dada ao modelo leúrico decorrente, a estrutura molecular, asscnte nas orbibais e configuraçòes clectrónicas dos elementos. Ao mudejo teórico é conferido uma realidade lísica muito mais concreta do que aquela que de facto possui.

E uma vez mais vêm à liça â. gunas das múltiplas querelas que o uso du modelo da esirulura molecular na construçăo da ciência química tem suscilado, ao longo dos anos. Delas ressallam, muito frequentementc, questōes muito sérias sobre a sua conciliaçắo cum a própria quítnica quântica. Púlo seu carácler dirccto e explícito, recordaremos aqui o interessamle rahalho de R.G. Woolley, já lá vàu mais de duas décadas, questionan. do logo no título o possivel "reaismo" da geometria molecular [22]. Vele, o auior mostra que a moderna concepçàn dume molćcula como un todo ligado por uma coleçăo du cluctrōes e núcleos não é invariaveluchue equivalenc ao modelo mulecular clássico de átomos mantidos juntos por ligaçoes. E daí conclui que a estrutura molecular não é uma propriedade in. trínseca das molciculas. Anos de. pois, este trabalbo de R.G.Woolley Ini objec1o de um outro hăo menos interessante da autoria de $\mathrm{S}$. J. Weininger que logo no título, rotula a estrutura mulecular como uп1 "quebra cahesas"[23]. Nele analisa a delicada problemática duma química quasc-dedutiva em que o formalismo quântico funçone como axioma.

A estrulura matemática da construçào da ciência da concepção galilaica, com raízes $\|$ platonismo. iornada paradigma da ciência moderna, levou a que uma abordagem dedutivo-presciente se tornasse paradigmática da mais elicaz explicação científica para a maioria dos físicos. Embora a fronteira que delimita os domínios das ciências química e lísica nem sempre seja marcada por contornos bem delinidos claros. é inegável que a ciência química tem um carácter c'xplicarivo é pragmático muitu mais acenluado que o da ciencia fisica onde a dedução e prediçầ résuliam meIhor [24]. Ora, se ma nrálica da própria ciência lisica há qự muito fundadamente, se oponha a uma abordagem marcadamente dedutiva, nāo surprecnde que a oposição ao mesmo lịpo de orientaçāo seja muito major cutre os cultores da ciência química |25|. A indefiniçăo, ou pior ainda, a confusāo. entre a realidade lógica e ontológica dos caracteres em quc está escrita a linguagem da natureza. só prejudica a posição assumida por чח e ollitos.

Porque a rcaliḑade lísica sc nào molda à rigidez de nuçôes matemalicas, a aplicação destas à sua descriçāo nāo esıá iscunla de situaçōes paradoxais que claramente indiciam a lalacidade da adequaçăo do lógico ao ontológico e justificam os limiles de preditabilidadt de qualquer modelo teórico.

As orbitais moleculares său clementos dum modelo logico cuja validade não innede que se pontha em causa o carácter ontolúgico dos elementos que o constituem, seja no seu todo, seja em algumas das suas partes. Heisenterg. um dos grandes obreiros dussc modelo, deixou-0 bem expresso, ao relerir-se à diliculdade subjacente ao alcgado paraúoxo da mecânica quầnlíca relativo à existência de nodos nas funçōes de onda gue descrevem as orbilais electrónicas. Aqui reproduzimos as suas palavras como conclusāo adequada às considideraçōes yuc lecemos a proposito da anunciada ohservação das orhitais d por M. J. Zuo c colaboradores:

"Essa dificuldado tem a ver cum a quesıào de as mais pequenas unidades da matéria serem ou não objectos [ísicos, existiren ou nãu do mesmo modo que existem as $\mathrm{pe}$ dras e as flores. Neste ponto, o desenvolvimerito da potia quântica mudou рor completo a situação. As lés de teoria quâuuica, formuladas matematicamente, mosiran claramente que os nossos habituais conceitos intuitivos nāu poden ser aplicadus. Todas as palavras e conceitos quc habitualmente usanos para descrever os ohjecios lísicus. tals como a posiçāo, a velocidade, a cor. etc., tornam-se indefinidas e problemáticas" [26].

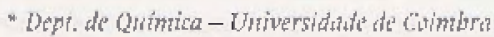
$3004-535$ dointso
}

\section{REFERÉNCIAS}

1. Calileo-Galilei, II Saggiatore, RRoma, 1623) in Opere, VI, p.232; c Leltre a Liceti de 11. ann.164t in Opere, XVt.l, p. 293. Nota: no prosente trabalho, as referéneclas als Obras de Galilieu remetem para os 21 volumes da edişo de A. Favaro i Florença. 1B90-1909) Opere Complete di Galileo Galilei

2. A. Koyré, Estudos Galilaicos, Publ. D. Qui xolc, lisboa, 1966, pp. $345 \mathrm{ss}$

3. Cialileo-Galilei, Did́logo soprat I che massimi sistemi del mondo (Florença, 16.32), 1 , in Opere. III. p.423

4. Ibidem. Opere, III, p. 424 .

5. A. Koyré, o.cit. p. 348 .

6. Calileo-Galilei, Diálogo stora I due massimi sutemi del mondo (Florença, 16.32), I in Opers, III, 1). 38 . 
7. A Kovré, o.cit... pp.349-351

8. J.M. Zuo, M. Kim, M. OKeefie e J.C. H Spence: Direct obsenation of d orbitals holes and $\mathrm{Cu}-\mathrm{Cu}$ bonding in Cu2O in Nature, 401 (1999), pp. $\$ 9-52$.

9. Colin 1. Humphreys, Electrons seen in orbit in Nature, 401 (1999), 21-22.

10. P, Zurer, Chem. Eng. News, 77148 [1999), p.39.

11. K. Leutwyler, Observing Orbitals in hlto:/www.sctam,com /explorations/1999/ 092099 cuprite.

12, M. lacoby. Chem. Eng. News, 77 (36) (1999), 8.

13. Ver, por exemplo: E. R. Scerri. Br. J. Phi105. 42 119911, 309-325; M.P. Melrose e E.R. Scerri. I. Chem. Educ. 73 (1996). 498-503; E. R. Scerri e L. Mclntyre. Synthese 111
11997,213-232; E. R. Sceri. Am. Sci. 85 (199/, 546-553; E.R. Scerri, Sci. Ant. 279 (19981, 78-83; E.R. Scerri, int. Stud. Philos.Sci, 1211998 1, 33-44; E.R. Scerri Foundations Chem, 2 12000), 1-4; E.R. Scerri, 1. Chem Educ. 77 12000), 522-525,

14. Eric R. Scerri, Have Orbitals Really Been Observed? in ). Chem. Educ., 77 (2000). 1492- 1494 .

\section{Loc, cit, pp. 1493-1494.}

16. M. A. Spackman, J.A.K. H loward and R Destro, Inf. Union Crwstal. Newlett, 8 [2000), 2.

17. S. G. Wang and W. H Schwary, Angew. Chem. Int. Ed. Engl. 39 (2000), 1757-1762.

18. J. F. Ogilvie, J. Chem. Educ, , 67 (1990), 280-289.

19. Loc. cit. p. 287
20. L. Pauling, f. Chen, Educ, , 69 , 1992: $519-521$

21. L. Pauling, ). Am. Chem. Soc, 33 (793) $1367-1400$

22. R.G. Woollev, Must a Molecule have a shape? in J. Am. Chen. Sor., 100119781. 1073-1078.

3. 5. I. Wenninger. The Moleculor Structure Conundrum: can Classical Chemistry be reduced to Quantum Chemistry? in f. Chemp. Educ., 6111984i, 939-944

24. D.W. Theobald, chem Soc. Rew.. 5 \{19761, 20$\}.$

25. D. Bohm, Brit. J. Phil. Sci., 12 |1961/62। 103.

26. W. Heisenberg. Across Frontiers, Harper \& Row, Nova lorquue, 1974, p. 114.

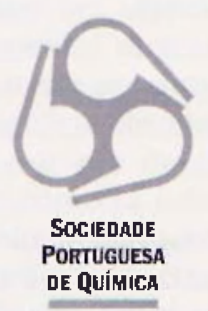

COLABORE

COM A

SOCIEDADE

\section{NÃO ATRASE $O$ PAGAMENTO DAS SUAS QUOTAS}

\section{SPQ - QUOTAS}

\section{Sócio Efectivo . . . . . . . . . . . . . . . 6 6000\$00 Sócio Estudante . . . . . . . . . . . . . . $3500 \$ 00$ Sócio Casal $\ldots \ldots \ldots \ldots \ldots \ldots \ldots \ldots \ldots$. . . . . 9000 \$ 00}

